Reprod. Nutr. Develop., 1988, 28 (3 B), 721-731

\title{
Déterminisme hormonal de la différenciation du muscle strié squelettique chez les amphibiens urodèles
}

\author{
C. CHANOINE, Chantal JANMOT (*), Michèle GUYOT-LENFANT, \\ J. P. DURAND $\left({ }^{* *}\right)$, Anne d'ALBIS $\left({ }^{*}\right)$, CI. L. GALLIEN
}

Laboratoire de Biologie du Développement, UA 1188 C.N.R.S.,

Université René Descartes, 45, rue des Saints-Pères, 75270 Paris Cedex 06.

(*) Laboratoire de Biologie Physicochimique, UA 1131 C.N.R.S., Bat. 433,

Université Paris-Sud, 91405 Orsay.

(**) Laboratoire souterrain du C.N.R.S., 09 Moulis.

Summary. Hormonal determinism of skeletal striated muscle differentiation in tailed amphibians.

In the urodelan amphibian Pleurodeles walt/ii, spontaneous external metamorphosis was correlated with an increase in the serum level of thyroxine (T4). Within the same period, a change occurred in the myofibrillar ATPase profile of the dorsal skeletal muscle : fibres of larval type were gradually replaced by transitional fibres (type IIC), then by adult fibres of the types I, IIA, and IIB. Likewise, a myosin isoenzymic transition was observed. In larval animals, myosin electrophoresis revealed 3 bands corresponding with isoforms having identical heavy chains (MHC), but different light chains (MLC). In the course of metamorphosis, the 3 larval isomyosins were replaced by 3 isoforms having the adult type $\mathrm{MHC}$ and different motility.

In a related neotenic species, Ambystoma mexicanum, no spontaneous anatomic metamorphosis occurred; at the time it should theoretically take place, the serum T4 level remained low. The ATPase profile was modified, but transitional fibres that replaced the initial larval types appeared to be persistent, and adult fiber types appeared only in a small amount. Myosin isoenzymic transition was also incomplete, larval isoforms were still distinguished in the neotenic adults.

Similar persistence of larval characters was observed in adult Proteus anguinus, a perennibranch that never undergoes anatomical metamorphosis.

Experimental hypothyroidian Pleurodeles walt/ii displayed no external metamorphosis, only the larval fibre types and isomyosins were detected in those animals.

External metamorphosis was induced in Ambystoma mexicanum by a triiodothyronine treatment. A complete myosin isoenzymic transition was observed in metamorphosed animals.

These results tend to indicate that a moderate increase in the level of thyroid hormones is sufficient to determine the production of the adult type MHC molecules and the differentiation of the corresponding myofibrillar types in the skeletal dorsal muscle of amphibians, while a marked increase would be necessary for repressing the initial larval feature.

\section{Introduction.}

Les hormones thyroïdiennes interviennent dans la régulation de nombreuses activités du métabolisme cellulaire (Hoch, 1974; Schwartz et Oppenheimer, 
1978 ; Menezes-Ferreira et Torresani, 1983). Chez les Vertébrés, elles jouent aussi un rôle important dans le déterminisme de divers phénomènes de différenciation cellulaire, au cours du développement et de la croissance de l'organisme (Greenberg et al., 1974 ; Cohen et al., 1978).

La métamorphose anatomique des amphibiens, en particulier, est directement corrélée à l'activité de la thyroïde (Etkin, 1968; Hourdry, 1980). Une augmentation du taux d'hormones thyroïdiennes circulantes durant cette période a été mise en évidence chez différentes espèces d'anoures (Leloup et Buscaglia, 1971 ; Miyauchi et al., 1977 ; Regard et al., 1978; Mondou et Kaltenbach, 1979), et d'urodèles (Eagelson et Mc Keown, 1978 ; Larras-Regard et al., 1981).

Chez l'urodèle néoténique Ambystoma mexicanum, différents travaux ont montré que l'absence de métamorphose anatomique résulte du faible niveau d'activité de la thyroïde observé chez ces animaux (Blount, 1950 ; Prahlad et de Lanney, 1965 ; Prahlad, 1968 ; Ituriza, 1971 ; Norris et Platt, 1973 ; Eagelson et Mc Keown, 1978). L'activité thyroïdienne, insuffisante pour provoquer une métamorphose complète, détermine cependant la modification de caractères biochimiques ou cytologiques (Charlemagne, 1967, 1972; Ouedraogo et Chalumeau-Le Foulgoc, 1973 ; Flavin et al., 1973; Ducibella, 1874a et b). Chez l'urodèle pérennibranche, Proteus anguinus, l'absence de métamorphose externe s'expliquerait en revanche par une sensibilité réduite des tissus cibles vis-à-vis du messager hormonal (Vialli, 1973 ; Schreiber, 1937 ; Durand, 1971).

Au total, la métamorphose peut être considérée comme un ensemble de différenciations contrôlées par les hormones thyroïdiennes, et susceptibles d'affecter divers tissus de l'organisme larvaire durant une phase bien définie de l'ontogenèse ; les modifications anatomiques externes qui l'accompagnent éventuellement ne représentent qu'un aspect du phénomène.

Dans ce travail nous nous proposons de démontrer que le taux d'hormones thyroïdiennes circulantes joue un rôle majeur dans le déterminisme de la différenciation terminale du muscle squelettique. Nous étudions les modifications du profil ATPasique myofibrillaire, et la transition des isoformes de la myosine dans le muscle strié dorsal de diverses espèces d'amphibiens urodèles, au cours du développement normal et dans des conditions expérimentales. L'effet de régulation exercé par le taux d'hormones thyroïdiennes circulantes sur la différenciation musculaire durant la métamorphose des amphibiens urodèles est mis en évidence.

\section{Matériel et méthodes.}

Animaux étudiés. -- Quatre espèces d'amphibiens urodèles sont utilisées dans cette étude: Pleurodeles waltlii Michahelles ( $P$. waltlii), Ambystoma mexicanum Shaw (A. mexicanum), Ambystoma tigrinum Green (A. tigrinum), et Proteus anguinus Laurenti ( $P$. anguinus).

Pour faciliter la comparaison entre ces espèces, la chronologie du développement de $A$. mexicanum et $P$. waltlii, est décrite par référence aux données établies pour cette dernière espèce par Gallien et Durocher (1957) ; suivant cette nomenclature la forme larvaire accomplie correspond au stade 54 . 
Chez $P$. waltiii la métamorphose anatomique débute spontanément chez les larves (stade 54) âgées de 90 à 100 jours. Elle s'achève au bout de 3 semaines, les animaux totalement métamorphosés sont alors au stade 56. Les larves de A. mexicanum et de $A$. tigrinum atteignent le stade 54 à l'âge de 100 à 120 jours. Les sujets de l'espèce $A$. tigrinum se métamorphosent spontanément, les individus de l'espèce $A$. mexicanum ne subissent pas de métamorphose externe. Chez tous ces animaux la maturité sexuelle est atteinte à l'âge de 1 à 2 ans, A. mexicanum se reproduisant sous sa forme larvaire.

Le développement de $P$. anguinus est lent. La phase embryonnaire correspond aux 115 premiers jours du développement, le développement larvaire se poursuit jusqu'au neuvième mois ; la larve présente ensuite une phase juvénile de longue durée et ne présente pas de métamorphose apparente. La maturité sexuelle n'est acquise qu'à l'âge de 12 à 14 ans (Durand, 1971).

Hypothyroïdie expérimentale. - Afin de prévenir l'activation de la thyroïde par la TSH, des embryons de $P$. waltlii au stade 22 sont hypophysectomisés par microchirurgie. Le développement de ces animaux aboutit à la formation de larves géantes qui ne se métamorphosent pas et demeurent bloquées au stade 54 . L'hypothyroïdie est aussi obtenue chez $P$. waltlii par l'addition journalière de thiourée $(0,3 / 1000)$ à l'eau des aquariums. Le traitement est appliquée à partir du stade 45 .

Métamorphose expérimentale. - Des larves de $P$. waltiii, âgées de 70 à 80 jours (stade 53-54), sont traitées par addition journalière à l'eau d'élevage de 3,5,3' triiodothyronine (T3) à la concentration de $5.10^{-8} \mathrm{M}$. Une métamorphose précoce est obtenue dans ces conditions. Le même traitement permet d'obtenir la métamorphose de sujets $A$. mexicanum.

Prélèvement des sérums. - Le sang est prélevé dans des tubes héparinés, par ponction cardiaque. Dans le cas des plus petites larves des échantillons provenant de plusieurs individus doivent être groupés de façon à obtenir, après centrifugation, des quantités suffisantes de sérum $(0,3$ à $0,5 \mathrm{ml})$. Les échantillons de sérum sont conservés à $-20^{\circ} \mathrm{C}$.

Prélèvement d'échantillons de musc/e. - Des échantillons de muscle dorsal (dorsalis trunci) sont prélevés par dissection. Les pièces destinés à l'analyse histochimique sont montées dans la gomme adragante, immergées dans l'isopentane et congelées dans l'azote liquide. Les échantillons réservés pour l'extraction de la myosine sont immédiatement congelés.

Dosage des hormones thyroidiennes. - La concentration de thyroxine (T4) dans les sérums est mesurée par un immunoessai spécifique (Immo Phase), modifié à partir du protocole recommandé par Corning Medical Laboratories.

Histochimie. - Des coupes transversales sériées $(8 \mu \mathrm{m})$ sont réalisées à partir des échantillons de muscle à l'aide d'un cryostat $\left(-22^{\circ} \mathrm{C}\right)$. Pour $P$. waltlii la méthode de coloration utilisée est celle décrite par Padykula et Hermann (1955), modifiée 
par Brooke et Kaiser (1969). La coloration des ATPases myofibrillaires est obtenue à $\mathrm{pH} 9,4$. Une préincubation de $10 \mathrm{~min}$ à la température ambiante est effectuée, soit à $\mathrm{pH}$ acide $(\mathrm{pH} \mathrm{4,63} \mathrm{et} \mathrm{pH} 4,35)$, soit à $\mathrm{pH}$ basique $(\mathrm{pH} \mathrm{9,4)}$. Pour $A$. mexicanum, $A$. tigrinum et $P$. anguinus, les meilleurs résultats sont obtenus dans les conditions expérimentales définies par Johnston et al. (1974) pour les poissons : la préincubation en milieu basique est effectuée à $\mathrm{pH} 10,4$ pendant 2 min. Les types de fibres sont décrits par référence à la nomenclature proposée par Brooke et Kaiser (1970) pour les mammifères. La distribution spatiale et le pourcentage des divers types de fibres ont été établis sur la surface totale de la section transversale du muscle, en étudiant 3 coupes provenant de 3 animaux différents pour chaque stade pris en considération.

Extraction de la myosine. - Les extraits sont préparés suivant la méthode de Pliszka et al. (1981).

Electrophorèse. - Les électrophorèses sont pratiquées suivant les procédures décrites par Hoh et al. (1976), et d'Albis et al. (1979). Les gels sont colorés par le bleu de Coomassie R 250, et les proportions relatives des différents isoenzymes sont établies à l'aide d'un densitomètre équipé d'un intégrateur. Deux à trois échantillons provenant d'animaux différents sont étudiés pour chaque stade du développement ou chaque type de traitement pris en considération.

\section{Résultats.}

Taux de thyroxine. - Chez $P$. waltlii la thyroxine (T4) n'est mise en évidence dans le sérum qu'à la fin du stade 54 ; sa concentration s'accroît régulièrement durant la métamorphose anatomique (stade 55). Après la métamorphose (stade 56) le taux de T4 diminue ; I'hormone ne peut plus être détectée chez les sujets adultes (planche I, fig. 1A). Chez A. mexicanum, la T4 n'est pas décelée dans les sérums d'animaux âgés de 2 et 3 mois. Sa concentration est faible dans les sérums de sujets âgés de 4 à 9 mois. L'hormone n'est plus mise en évidence chez les individus plus âgés (planche l, fig. 1B).

Profil ATPasique myofibrillaire. - Chez $P$. waltlii aux stades larvaires 53 et 54 , l'ensemble des fibres musculaires est fortement coloré après la préincubation à $\mathrm{pH} 9,4$; la réaction est inversée lorsque la préincubation est effectuée en milieu acide ( $\mathrm{pH} \mathrm{4,63} \mathrm{et} \mathrm{4,35).} \mathrm{Au} \mathrm{plan} \mathrm{de} \mathrm{l'activité} \mathrm{ATPasique} \mathrm{toutes} \mathrm{les} \mathrm{fibres}$ appartiennent donc au stade 54 à un unique type "larvaire ». Toutefois, il est possible de distinguer 2 populations de fibres, qui diffèrent par leur taille et leur distribution topographique. La zone périphérique du muscle, sous la peau, est formée par des fibres de faible diamètre; la région centrale présente un mélange de fibres de petite taille et de grande taille, lui conférant un aspect particulier en mosaïque. Aux stades $55 a$ et $55 b$ toutes les fibres ont encore une coloration intense après la préincubation à $\mathrm{pH} \mathrm{9,4;} \mathrm{après} \mathrm{une} \mathrm{préincubation} \mathrm{à} \mathrm{l'un} \mathrm{ou} \mathrm{l'autre}$ des $2 \mathrm{pH}$ acides les petites fibres ( $70 \%$ du nombre total de fibres) sont encore 
colorées, alors que les fibres de plus grand diamètre (30\%) ne montrent pratiquement aucune coloration. Au stade $55 \mathrm{c}$ l'activité ATPasique après préincubation au $\mathrm{pH}$ basique révèle $90 \%$ de fibres fortement colorées, de grand diamètre ou de taille intermédiaire, se rapportant au type $\mathrm{II}$, et $10 \%$ de petites fibres faiblement colorées classées dans le type I. La réaction est inversée lorsque la préincubation est faite à $\mathrm{pH}$ acide : les fibres de types I ont une coloration intense. $\mathrm{A} \mathrm{pH} \mathrm{4,63} \mathrm{les} \mathrm{plus} \mathrm{grandes} \mathrm{fibres} \mathrm{de} \mathrm{type} \mathrm{II} \mathrm{sont} \mathrm{totalement} \mathrm{dépourvues}$ de coloration, elles sont de type IIA ( $20 \%$ du total des fibres). Les plus petites fibres de type II, encore colorées à $\mathrm{pH} 4,63$, apparaissent très claires à $\mathrm{pH} 4,35$ et peuvent être classées dans les types IIB et/ou IIC (70\% du total des fibres). Chez l'adulte on distingue $13 \%$ de fibres de type I, et $87 \%$ de fibres de type II. La proportion des fibres IIA est sensiblement accrue $(67 \%$ du nombre total de fibres). Les autres fibres de type II (20\% des fibres) peuvent être clairement rapportées au type IIB : en effet elles sont totalement claires après une préincubation à $\mathrm{pH} 4,35$. On n'observe plus de fibres $\mathrm{IIC}$.

Chez $A$. mexicanum, au stade 53-54 (animaux âgés de 4 mois), les fibres sont du type "larvaire ». On retrouve les deux populations déjà décrites chez $P$. waltlii. Chez les sujets âgés de 7 mois, les grandes fibres (18\% du nombre total) ont une coloration assez faible pour les deux $\mathrm{pH}$ acides de préincubation, alors que les fibres de petite taille ( $82 \%$ du total des fibres) se colorent plus intensément, de façon hétérogène. Après le $8^{\mathrm{e}}$ mois, 3 types de fibres sont identifiés. La préincubation à $\mathrm{pH}$ basique $(\mathrm{pH} 10,4)$ indique que certaines des petites fibres, situées en position périphérique, sous la peau ou autour des côtes appartiennent au type $\mathrm{I}$. Cette interprétation est confirmée après la préincubation aux deux $\mathrm{pH}$ acides $(4,63$ et 4,35$)$; une seconde population de fibres de faible diamètre appartient au type IIB. Les autres fibres, grandes et petites, se rapportent au type IIC. Chez les animaux âgés de 11 mois les proportions respectives des différents types de fibres sont les suivantes : $9 \%$ de type I, $18 \%$ de type IIB, et $73 \%$ de type IIC. Aucune fibre de type IIA n'est encore observée à ce stade ; les premières fibres de type IIA, qui sont de grande taille, apparaissent dans les tissus de sujets âgés de 12 mois.

Quatre types de fibres sont mis en évidence chez les adultes néoténiques âgés de 3 ans : type I (14\%), type IIA (10\%), type IIB (16\%) et type IIC (60\%). La persistance d'un nombre important de fibres IIC dans le muscle de l'adulte non métamorphosé de $A$. mexicanum doit être soulignée, la situation est en effet différente chez des adultes de même âge appartenant à l'espèce voisine A. tigrinum, qui ont subi une métamorphose anatomique. On ne retrouve chez ces animaux que 3 types de fibres : type I (17\%), type IIA (57\%) et type IIB (26\%).

Chez $P$. anguinus, au $3^{\mathrm{e}}$ mois du développement, les fibres sont toutes du type " larvaire ». Chez les sujets âgés de 4 ans, les réactions ATPasiques indiquent I'existence de fibres de type IIB qui représentent $10 \%$ de la population totale. Les autres fibres appartiennent au type I et au type IIC. Les petites fibres de type I (28\%) sont claires après la préincubation à $\mathrm{pH} \mathrm{10,4;} \mathrm{cependant} \mathrm{la} \mathrm{réaction} \mathrm{n'est}$ pas inversée aux $\mathrm{pH}$ acides de préincubation ce qui ne permet pas de les différencier de façon absolue des fibres IIC (62\%). Les fibres de type IIA sont mises en évidence chez les individus âgés de 6 ans ; à ce stade elles représentent seulement $1 \%$ du nombre total de fibres. 
Chez les adultes non métamorphosés âgés de 16 ans, les proportions sont les suivantes: types I et/ou IIC $67 \%$ (respectivement $15 \%$ «type I» et $52 \%$ type IIC), type IIB $29 \%$, type IIA $4 \%$; la baisse relative du pourcentage de fibres IIC corrélée à l'accroissement du nombre de fibres de type IIA et IIB doit être notée.

Transition isoenzymique de la myosine. - Chez $P$. walt/ii l'analyse électrophorétique de la myosine extraite du muscle dorsal jusqu'au stade 54 montre 3 bandes distinctes correspondant à des isoformes "larvaires ». Au stade 55 , durant la métamorphose anatomique, de nouvelles bandes de mobilité plus faible font leur apparition; 9 bandes au moins peuvent être décelées au stade $55 \mathrm{c}$. Après la métamorphose les isoformes de mobilité électrophorétique intermédiaire, puis les isomyosines larvaires disparaissent, si bien que chez l'adulte seules les 3 bandes présentant la plus faible mobilité sont encore observées (planchel, fig. 1A). Chez A. mexicanum, les sujets âgés de moins de 4 mois montrent 3 isomyosines de même mobilité électrophorétique que les bandes larvaires de $P$. walt/ii. De nouvelles bandes de mobilité plus faible apparaissent progressivement dans la suite du développement, mais les 3 isomyosines de type larvaire persistent et sont toujours décelées chez les sujets adultes non métamorphosés (planche I, fig. 1B). Par contraste, l'analyse électrophorétique de la myosine extraite du muscle dorsal de sujets adultes métamorphosés de $A$. tigrinum montre l'existence de 3 bandes seulement, dont la mobilité correspond à celle des isomyosines adultes de $P$. waltlii. Chez $P$, anguinus âgé de 3 mois, trois isomyosines de type larvaire sont mises en évidence. Chez les sujets âgés de 4, 6 et 16 ans de nouvelles bandes de mobilité plus faible sont observées, mais les isoformes larvaires sont toujours présentes (planchel, fig. 2).

\section{PLANCHE I}

FIG. 1. - Taux de thyroxine (nmol./I) dans les sérums et polymorphisme des myosines natives (électrophorèse dans les conditions non dissociantes) d'extraits de muscle dorsal d'amphibiens urodèles. La proportion ( $\pm 5 \%$ ) des myosines de type adulte à chaque stade du développement est indiquée sous le gel correspondant. A : $P$. waltlii. Les chiffres correspondent aux différents stades du développement. Stades 42 et 54 : prométamorphose. Stades $55 \mathrm{~b}$ et $55 \mathrm{c}$ : métamorphose. Stade $56 \mathrm{a}, \mathrm{b}, \mathrm{c}$ : post-métamorphose (post.). Ad: Adulte. B : A. mexicanum. Les chiffres indiquent l'âge de l'animal, de 4 mois à 2 ans.

FIG. 2. - Polymorphisme des myosines natives (électrophorèse dans les conditions non dissociantes) d'extraits de muscle dorsal de $\mathrm{P}$. anguinus. Les chiffres indiquent l'âge de l'animal, de 3 mois à 16 ans.

FIG. 3. - Electrophorèses dans les conditions non dissociantes de myosines natives extraites du muscle dorsal de sujets hypothyroidiens de l'espèce $\mathrm{P}$. waltlii. \% représente le pourcentage de myosines adultes. A : Animaux âgés de 105 jours. $H=$ Sujet hypophysectomisé ; $T=$ Témoin en cours de métamorphose. $\mathrm{B}$ : Animaux âgés de 120 jours. $\mathrm{H}=$ Sujet hypophysectomisé ; $T$ = Témoin métamorphosé. L.G. = Larve géante obtenue par un traitement à la thiourée, et dont le traitement est interrompu depuis 10 jours. $\mathrm{C}$ : Animaux âgés de 130 jours. $\mathrm{H}=$ Sujet hypophysectomisé ; $\mathrm{T}=$ Témoin métamorphosé. D : Animaux âgés de 14 mois. L.G. = Larve géante obtenue par un traitement à la thiourée; $\mathrm{T}=$ Témoin métamorphosé

FIG. 4. - Electrophorèses dans des conditions non dissociantes de myosines extraites du muscle dorsal de A. mexicanum après métamorphose anatomique expérimentale. Les animaux ont été traités à la T3 pendant 3 semaines à l'âge de 5 mois. A : Animaux âgés de 7 mois. T3 $=$ Sujet métamorphosé depuis 5 semaines; $T=$ Témoin non métamorphosé. $B$ : Animaux âgés de 18 mois. T3 = Sujet métamorphosé depuis 12 mois ; $T=$ Témoin non métamorphosé. 


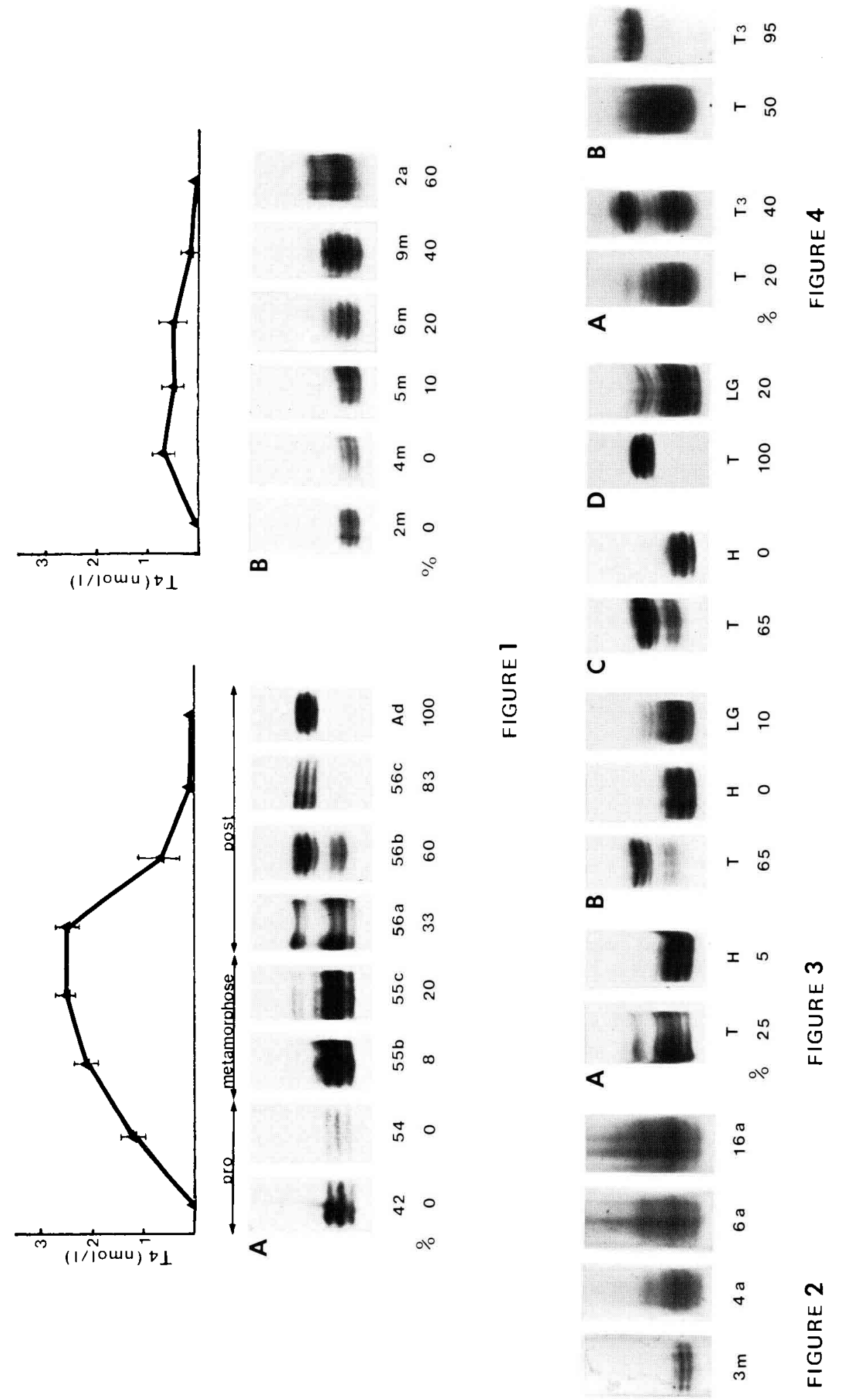


Hypothyroïdisme. - Chez $P$. waltlii les sujets qui ont été hypophysectomisés au stade 22 ne subissent pas de métamorphose anatomique ; le taux de T4 sérique de ces animaux est toujours inférieur au seuil de sensibilité de la méthode de dosage utilisée. Chez les animaux hypophysectomisés âgés de 130 jours, l'étude du profil ATPasique myofibrillaire du muscle dorsal révèle seulement la présence de fibres de type "larvaire ». La myosine analysée par électrophorèse, ne montre que les 3 bandes de type larvaire (planche l, fig. 3). Chez les larves géantes de $P$. waltlii âgées de 6 à 9 mois, obtenues par le traitement à la thiourée, le profil ATPasique myofibrillaire et la distribution électrophorétique des isoformes de la myosine sont aussi ceux que l'on observe normalement chez les témoins âgés de 3 mois (stade 54). En revanche, chez un individu traité âgé de 14 mois et ne montrant aucun signe de métamorphose, le profil ATPasique correspond à celui d'un témoin au stade $55 \mathrm{c}$, et l'électrophorèse de la myosine permet de déceler, en plus des 3 isomyosines larvaires, des bandes faiblement marquées de type intermédiaire ou adulte.

Métamorphose expérimentale. - Chez $P$. walt lii des larves au stade 53 sont traitées par la T3; ces animaux atteignent le stade 56 au bout de 3 semaines, et la transition isoenzymique de la myosine est accélérée de façon significative par rapport aux témoins, qui sont encore au stade 55a. Des sujets de l'espèce A. mexicanum âgés de 5 mois, traités par la T3, subissent la métamorphose anatomique. L'analyse électrophorétique de la myosine un mois après la fin du traitement témoigne du renforcement des bandes de faible mobilité et d'une diminution de l'intensité des bandes de type larvaires. Un an après la métamorphose, seules les bandes de faible mobilité correspondant aux isomyosines adultes sont mises en évidence, la transition isoenzymique de la myosine étant alors complète (planche I, fig. 4).

\section{Discussion.}

Les résultats présentés dans ce travail montrent qu'il existe, chez les amphibiens urodèles, une corrélation entre l'accroissement du taux des hormones thyroïdiennes circulantes et la métamorphose. En particulier le déterminisme hormonal de la différenciation terminale du muscle strié squelettique dorsal, qui constitue un des aspects de la métamorphose est mis en évidence.

Lorsque la concentration d'hormones thyroïdiennes circulante augmente rapidement et de façon sensible, que ce soit spontanément ( $P$. waltiii et A. tigrinum), ou à la suite d'interventions expérimentales (A. mexicanum), la métamorphose anatomique se produit en 3 semaines et la différenciation complète du muscle se poursuit jusqu'à son terme. Quand le taux d'hormones thyroïdiennes est naturellement peu élevé (A. mexicanum), ou qu'il est expérimentalement maintenu à un faible niveau ( $P$. walttii), la métamorphose externe n'est pas observée. Dans ce cas cependant, si la différenciation du muscle reste incomplète, elle s'effectue de façon partielle. Une différenciation partielle du muscle dorsal se produit également chez le pérennibranche $P$. anguinus au cours de la phase juvénile de l'ontogenèse, ce qui montre que chez cette espèce aussi certains tissus 
sont aptes à engager effectivement un phénomène de métamorphose. II existe une étroite relation entre l'évolution du profil ATPasique myofibrillaire et la transition isoenzymique de la myosine. Dans le muscle dorsal de $P$. waltlii, 4 types de fibres sont identifiés chez les larves en cours de la métamorphose : I, IIA, IIB et IIC. Finalement, seuls les types I, IIA et IIB sont retrouvés chez l'adulte. Parallèlement on observe le remplacement progressif de 3 isomyosines larvaires par 3 isoenzymes adultes. La même situation est observée chez les adultes métamorphosés de $A$. tigrinum. Par contraste, dans les espèces $A$. mexicanum et $P$. anguinus les fibres de type IIC demeurent nombreuses chez les adultes non métamorphosés, alors que la proportion des fibres de type $\mathrm{A}$ est faible. De la même façon on note sur les électrophorèses correspondant à la myosine de ces animaux, la persistance des isomyosines larvaires et des bandes intermédiaires.

Ainsi, chez les amphibiens urodèles comme chez les mammifères (PierobonBormioli et al., 1981), il apparaît que les fibres de type IIC pourraient être des fibres de transition, contenant à la fois des isomyosines adultes et des isomyosines larvaires.

Les sujets $P$. waltlii rendus expérimentalement hypothyroïdiens conservent le profil ATPasique myofibrillaire et la constitution isoenzymique de la myosine du type larvaire. D'autre part l'administration de triiodothyronine accélère la transition isoenzymique de la myosine chez $P$. walt/ii, et permet la poursuite d'une évolution complète de cette transition chez $A$. mexicanum. Ceci indique bien que chez les amphibiens urodèles, la transition isoenzymique de la myosine dans le muscle squelettique dorsal est contrôlée par les hormones thyroïdiennes.

Une faible concentration d'hormones suffit à déterminer la production des isomyosines adultes, mais une concentration plus élevée est nécessaire pour réduire ou interrompre la production des isomyosines larvaires. Les isomyosines larvaires et les isomyosines adultes du muscle squelettique dorsal des amphibiens urodèles possèdent les mêmes types de chaînes légères (MLC), mais des chaînes lourdes (MHC) différentes (d'Albis et al., 1985b). Par conséquent les modifications déterminées par les variations du taux d'hormones thyroïdiennes ne concernent que les chaînes lourdes de la myosine. Les phénomènes observés au niveau du muscle squelettique chez les amphibiens urodèles lors de la métamorphose, sont donc à rapprocher de ceux qui sont mis en évidence chez la souris dans la période post-natale (d'Albis et al., 1985a, c ; Whalen et al., 1985), et on peut penser qu'un système génétique voisin de celui mis en évidence chez la souris par Weydert et al. $(1983,1985)$ règle la différenciation terminale du muscle strié squelettique des amphibiens urodèles, sous le contrôle des hormones thyroïdiennes.

$13^{\circ}$ Réunion du groupe Développement I.N.R.A., Cap d'Adge, 25-27 mai 1987.

\section{Références}

BLOUNT R. F., 1950. The effects of heteroplastic hypophysial grafts upon the axolotl, Ambystoma mexicanum. J. exp. Zool., 113, 717-739. 
BROOKE M. H., KAISER K. K., 1969. Some comments on the histochemical characterization of muscle adenosine triphosphatase. J. Histochem. Cytochem., 17, 431-432.

BROOKE M. H., KAISER K. K., 1970. Muscle fiber types. How many and what kind. Arch. Neurol., 25, 369-379.

CHARLEMAGNE J., 1967. Etude par électrophorèse et immunoélectrophorèse des protéines sériques de I'Axolotl (Ambystoma mexicanum Shaw). Bull. Soc. Zool. Fr., 92, 739-748.

CHARLEMAGNE J., 1972. Aspects morphologiques de la différenciation des éléments sanguins chez l'Axolotl, Ambystoma mexicanum Shaw. Z. Zellforsch. Mikrosk. Anat., 123, 224-239.

COHEN P. P., BRUCKER R. F., MORRIS S. M., 1978. Cellular and molecular aspects of thyroid hormone action during amphibian metamorphosis. In LI C. H., Hormonal proteins and peptides, 6, 273-381, Acad. Press, New York.

D'ALBIS A., CHANOINE C., JANMOT C., WEINMAN J., GALLIEN CI. L., 1985a. Variations du taux des hormones thyroidiennes plasmatiques et différenciation du muscle squelettique au cours de la période périnatale chez la souris. Arch. Anat. microsc., 74, 251.

D'ALBIS A., JANMOT C., BÉCHET J. J., 1985b. Myosin switches in skeletal muscle development of an urodelan amphibian, Pleurodeles walthii. Comparison with a mammalian, Mus musculus. Biochem. biophys. Res. Comm., 128, 94-100.

D'ALBIS A., JANMOT C., WEINMAN J., GALLIEN CI. L., 1985c. Myosin isoenzymic modification at critical stages of development of mice and urodelan amphibians, 667-674. In E. E. ALIA, N. ARENA, M. A. RUSSO, Contractile proteins in muscle and nonmuscle cell systems. Biochemistry, physiology and pathology. Praeger, New York.

D'ALBIS A., PANTALONI C., BECHET J. J., 1979. An electrophoretic study of native myosin isozymes and of their subunit content. Eur. J. Biochem., 99, 261-272.

DUCIBELLA T., 1974a. The occurrence of biochemical metamorphic events without anatomical metamorphosis in the axolotl. Dev. Biol., 38, 175-186.

DUCIBELLA T., 1974b. The influence of L-thyroxine on the change in red blood cell type in the axolotl. Dev. Biol., 38, 187-194

DURAND J. P., 1971. Recherches sur l'appareil visuel du protée, Proteus anguinus Laurenti, Urodèle hypogé. Thèse $\mathrm{n}^{\circ} 460$, Univ. P. Sabatier, Toulouse.

EAGELSON G. W., MCKEOWN B. A., 1978. Changes in thyroid activity of Ambystoma gracile (Baird) during different larval, transforming, and postmetamorphic phases. Can. J. Zool., 56, 1377-1381.

ETKIN W., 1968. Hormonal control of amphibian metamorphosis, 313-348. In W. ETKIN, L. I. GILBERT, Metamorphosis a problem in developmental biology. Appelton-Century Crofts, New York.

FLAVIN M., GASSER F., BEETSCHEN J. C., 1973. Influence de l'accélération ou de l'inhibition expérimentales de la métamorphose sur l'évolution des hémoglobines chez Pleurodeles waltlii Michah. (Amphibien, Urodèle). C.R. Acad. Sci. Paris, 277, 2773-2776.

GALLIEN L., DUROCHER M., 1957. Table chronologique du développement chez Pleurodeles waltlii Michah. Bull. Biol. Fr. Belg., 19, 97-114.

GREENBERG A. H., NAJJAR S., BLIZZARD R. M., 1974. Effect of thyroid hormone on growth, differentiation and development. In GREER M. A., SOLOMON D. H., Handbook of Physiology. Am. physiol. Soc., Washington D.C., 3, 377-389.

HOCH I. L., 1974. Metabolic effects of thyroid hormones. In GREER M. A., SOLOMON D. H., Handbook of physiology. Am. physiol. Soc., Washington D.C., 3, 391-411.

HOH J. F. Y., McGRATH P. A., WHITE R. I., 1976. Electrophoretic analysis of multiple forms of myosin in fast twitch and slow twitch muscles of the chick. Biochem. J., 157, 87-95.

HOURDRY J., 1980. Quelques aspects biochimiques de la métamorphose des Amphibiens. Ann. Biol., 19, 250-287.

ITURIZA F. C., 1971. Neurosecretion in the median eminence of the axolotl after induction of metamorphosis. Neuroendocrinology, 7, 156-163.

JOHNSTON I. A., PATTERSON S., WARD P., GOLDSPINK G., 1974. The histochemical demonstration of myofibrillar adenosine triphosphatase activity in fish muscle. Can. J. Zool., 52, 871-877.

LARRAS-REgARD E., TAUROG A., DORRIS M., 1981. Plasma T4 and T3 levels in Ambystoma tigrinum at various stages of metamorphosis. Gen. Comp. Endocrinol., 43, 443-450. 
LELOUP J., BUSCAGLIA M., 1971. La triiodothyronine, hormone de la métamorphose des Amphibiens. C.R. Acad. Sci. Paris. 284, 2261 - 2263.

MENEZES-FERREIRA M. M., TORRESANI J., 1983. Mécanismes d'action des hormones thyroïdiennes au niveau cellulaire. Ann. Endocrinol., 44, 205-216.

MIYAUCHI H., LA ROCHELLE F. T. Jr., SUZUKI M., FREEMAN M., FRIEDEN E., 1977. Studies on thyroid hormones and their binding in bullfrog tadpole plasma during metamorphosis. Gen. comp. Endocrinol., 33, 254-266.

MONDOU P. M., KALTENBACH J. C., 1979. Thyroxine concentrations in blood serum and pericardial fluid of metamorphosing tadpoles and of adult frogs. Gen. comp. Endocrinol., 39. 343-349.

NORRIS D. O., PLATT J. E., 1973. Effects of pituitary hormones, melatonin, and thyroid inhibitors on radioiodide uptake by the thyroid glands of larval and adult tiger salamanders, Ambystoma tigrinum (Amphibia : Caudata). Gen. comp. Endocrinol., 21, 368-376.

OUEDRAOGO L., CHALUMEAU-LE FOULGOC M. Th., 1973. Influence de la métamorphose expérimentale sur les isozymes de la déshydrogénase lactique du plasma et des hématies, chez Ambystoma mexicanum Shaw. C. R. Acad. Sci. Paris, 276, 1749-1752.

PADYKULA H. A., HERMAN E., 1955. The specificity of the histochemical method of adenosine triphosphatase. J. Histochem. Cytochem., 17, 170-195.

PIEROBON-BORMIOLI S., SARTORE S., DALLA LIBERA L., VITADELLO M., SCHIAFFINO S., 1981. "Fst" isomyosins and fiber types in mammalian skeletal muscle. J. Histochem. Cytochem., 29, 1179-1188.

PLISZKA B., STRZELECKA-GOLASZEWSKA H., PANTALONI C., D'ALBIS A., 1981. Comparison of myosin isoenzymes from slow-tonic and fast-twitch fibers of frog muscle. Eur. J. Cel/ Biol., 25, 144-149.

PRAHLAD K. V., 1968. Induced metamorphosis: rectification of a genetic disability by thyroid hormone in the mexican axolotl Siredon mexicanum. Gen. comp. Endocrinol., 11, 21-30.

PRAHLAD K. V., DE LANNEY L. E., 1965. A study of induced metamorphosis in the axolotl. J. exp. Zool., 160, 137-146.

REGARD E., TAUROG A., NAKASHIMA T., 1978. Plasma thyroxine and triiodothyronine levels in spontaneously metamorphosing Rana catesbeiana tadpoles and in adult anuran Amphibia. Endocrinology, 102, 674-684.

SCHREIBER G., 1937. Ricerche sulla metamorfosi degli innesti xenoplastici di pelle di Urodele neotenici. Monitore Zool. ital., 47, suppl., 179-181.

SCHWARTZ H. L., OPPENHEIMER J. H., 1978. Physiologic and biochemical actions of thyroid hormone. Pharmac. Ter., 3, 349-376.

VIALLI M., 1931. Ricerche sulla metamorfosi degli anfibi - 1 Innesti di tiroide di Triton alpestris neotenico e di Proteus anguineus. Boll. Soc. Ital. Biol. sper., 6, 1049-1059.

WEYDERT A., DAUBAS P., CARAVATTI M., MINTY A., BUGAISKY G., COHEN A., ROBERT B., BUCKINGHAM M., 1983. Sequential accumulation of mRNA encoding different myosin heavy chain isoforms during skeletal muscle development in vivo detected with a recombinant plasmid identified as coding for an adult fast myosin heavy chain from mouse skeletal muscle. J. biol. Chem., 258, 13867-13874.

WEYDERT A., DAUBAS P., LAZARIDIS I., BARTON P., LEADER D. P., BONHOMME F., CATALAN J., SIMON D., GUENET J. L., GROS F., BUCKINGHAM M., 1985. Genes for skeletal muscle myosin heavy chains are clustered and are not located on the same mouse chromosome as cardiac myosin heavy chain gene. Proc. nat. Acad. Sci. USA, 82, 7183-7187.

WHALEN R. G., TOUTANT M., BUTLER-BROWNE G. S., WATKINS S. C., 1985 . Hereditary pituitary dwarfism in mice affects skeletal and cardiac myosin isozyme transitions differently. J. Cell Biol., 101, 603-609. 